\title{
Food Frequency Questionnaires: Are they appropriate for foods that are consumed irregularly, such as eggs?
}

\author{
Emmy van den Heuvel, Jane L. Murphy and Katherine M. Appleton \\ Bournemouth University, Bournemouth, United Kingdom
}

\begin{abstract}
Eggs tend to be eaten irregularly, e.g. they are often eaten as a standby for unplanned meals, and have been reported to be eaten as a treat or on special occasions. This intrapersonal variation may impact the accuracy of measuring the habitual intake of eggs. Food frequency questionnaires (FFQs) are often validated for nutrients but not foods, and may not be suitable for measuring the consumption of individual foods. The validity of measuring the consumption of specific foods can be particularly affected for foods with a greater within-person variation. Moreover, FFQs rarely include a detailed intake measure of eggs, and often do not include eggs in mixed dishes. With epidemiological studies focussing on individual foods to assess associations between foods and diseases, it is important to consider the accuracy of dietary assessment methods in measuring intake of individual foods.

In the current study we compare egg intake data from a validated FFQ and egg intake data from an FFQ specifically designed to measure egg intake. Both questionnaires were completed by a sample of 100 community-dwelling healthy older adults, which included 54 females and 46 males, with mean age $70(\mathrm{SD}=7)$ years. Both FFQs had a similar layout, but the validated FFQ section on egg intake consisted of three questions on egg intake, while the egg FFQ included 18 preparations of eggs, including mixed dishes.

Mean monthly egg consumption for the validated FFQ was $16(\mathrm{SD}=13)$ eggs, while the egg FFQ showed an egg intake of 22 $(\mathrm{SD}=16)$ eggs per month, suggesting under- or over-reporting on one or both measures. Although the mean values are noticeably different, validated FFQ egg intake correlates strongly with egg FFQ intake $(r=.773, p<.001)$.

National Diet and Nutrition Survey data indicates that British older adults ( 65 years + ) consume $33 \mathrm{~g}$ of eggs/egg dishes per day, the equivalent of 16-17 eggs per month. This means that the validated FFQ egg intake of 16 eggs per month is more similar to the NDNS data. However, FFQs generally tend to overestimate food intake compared to diet records and the validated FFQ is validated for the total nutrient intake, not for individual foods. With only three questions and without including mixed dishes, the validated FFQ may under-estimate intake of eggs, while the egg FFQ may be more comprehensive. More research is needed to explore the most appropriate methods for measuring intakes of foods that are irregularly consumed, such as eggs.
\end{abstract}

\section{Conflict of Interest}

There is no conflict of interest 